\title{
Serine/threonine kinase 15 gene polymorphism and risk of digestive system cancers: A meta-analysis
}

\author{
JIANFEI LUO, RUICHENG YAN and LI ZOU
}

\begin{abstract}
Department of Gastrointestinal Surgery, Renmin Hospital of Wuhan University, Wuhan, Hubei 430060, P.R. China
\end{abstract}
Received February 28, 2014; Accepted November 3, 2014

DOI: $10.3892 /$ etm. 2014.2070

\begin{abstract}
Previous studies have reported an association between the two coding polymorphisms (91T $>$ A and $169 \mathrm{G}>\mathrm{A}$ ) of the serine/threonine kinase 15 (STK15) gene and the risk of digestive system cancers; however, the results are inconsistent. In the present study, a meta-analysis was carried out to assess the association between the two STK15 polymorphisms and the risk of digestive system cancers. Relevant studies were identified using PubMed, Web of Science, China National Knowledge Infrastructure, WanFang and VIP databases up to February 18, 2014. The pooled odds ratio (OR) with a $95 \%$ confidence interval (CI) was calculated using the fixed or random effects model. A total of 15 case-control studies from 14 publications were included. Of these, 15 studies concerned the $91 \mathrm{~T}>\mathrm{A}$ polymorphism and included 7,619 cases and 7,196 controls and four studies concerned the $161 \mathrm{G}>\mathrm{A}$ polymorphism and included 826 cases and 713 controls. A significantly increased risk of digestive system cancers was observed for the $91 \mathrm{~T}>\mathrm{A}$ polymorphism (recessive model: OR, 1.19; 95\% CI, 1.07-1.31). In subgroup analysis by ethnicity, a significant association was detected in Asian populations (recessive model: OR, 1.21; 95\% CI, 1.08-1.36) but not in Caucasian and mixed populations. Stratification by tumor type indicated that the 91T $>$ A polymorphism was associated with an increased risk of esophageal and colorectal cancers under the recessive model (OR, 1.19; 95\% CI, 1.03-1.38; and OR, 1.24; 95\% CI, 1.04-1.46; respectively); however, no significant association was observed between the $169 \mathrm{G}>\mathrm{A}$ polymorphism and the risk of digestive system cancers in any of the genetic models. Furthermore, in subgroup analysis by ethnicity, similar results were observed in the Asian and Caucasian populations. The present meta-analysis demonstrated that the STK15 gene $91 \mathrm{~T}>\mathrm{A}$ polymorphism, but not the $169 \mathrm{G}>\mathrm{A}$ polymorphism,
\end{abstract}

Correspondence to: Professor Jianfei Luo, Department of Gastrointestinal Surgery, Renmin Hospital of Wuhan University, 238 Jiefang road, Wuhan, Hubei 430060, P.R. China

E-mail: ljfwuhan@163.com

Key words: serine/threonine kinase 15, polymorphism, digestive system cancers, meta-analysis may be a risk factor for digestive system cancers, particularly for esophageal and colorectal cancers.

\section{Introduction}

Digestive system cancers, including esophageal, gastric, hepatocellular, bowel, pancreatic, gallbladder and anal cancers, are the most common types of cancer worldwide. There are an estimated 3.4 million new cases diagnosed worldwide each year and the mortality rates have increased over the past decade (1). Although the exact mechanism of carcinogenesis remains to be fully understood, accumulating evidence has confirmed that certain risk factors (such as dietary, ethnic and socioeconomic factors) and interactions between genetic and environmental factors may play important roles in the pathogenesis of these types of cancer $(2,3)$.

Serine/threonine kinase 15 (STK15, also known as Aurora-A or AURKA) is a centrosome-localized serine/threonine kinase that is involved in cell cycle regulation, particularly the passage from $\mathrm{G}_{2}$ to $\mathrm{M}$, through the formation of mitotic spindles (4). The STK15 gene, which consists of nine exons, is located on chromosome $20 \mathrm{q} 13.2$, a region frequently amplified and overexpressed in various types of human cancer (5). STK15 has been reported to be overexpressed in numerous types of malignancies, including colorectal and pancreatic cancers $(6,7)$. Considerable evidence indicates that overexpression of the STK15 gene results in centrosome amplification, chromosomal instability, aneuploidy and transformation (8). Two non-synonymous polymorphisms, 91T >A (rs2273535) and 169G $>$ A (rs1047972), have been identified in the STK15 gene. A thymine (T)/adenine (A) polymorphism located at nucleotide position 91 encodes a phenylalanine (Phe)-to-isoleucine (Ile) substitution at amino acid position 31. A guanine (G)/A polymorphism at nucleotide 169 encodes a valine (Val)-to-Ile substitution at amino acid position 57 . The two polymorphisms are located within two conserved motifs in the $\mathrm{N}$-terminus region of the STK15 gene (9). It has been revealed that the A allele of the $91 \mathrm{~T}>\mathrm{A}$ (31Ile $>$ Phe) polymorphism is preferentially amplified and more potent than the $\mathrm{T}$ allele in leading to aneuploidy and transformation (8). Furthermore, the 169G >A (57 Val>Ile) polymorphism was found to affect the kinase activity of aurora kinase A (10).

Studies have suggested the presence of an association between the two coding polymorphisms in the STK15 gene and an increased risk of digestive system cancers (10-23); 
however, the results have been inconsistent. The aim of the present study was therefore to conduct a meta-analysis to evaluate the association between the two STK15 polymorphisms and susceptibility to digestive system cancers.

\section{Materials and methods}

Search strategy. The electronic literature databases of PubMed, Web of Science, China National Knowledge Infrastructure (CNKI), WanFang and VIP were searched for all relevant articles. The last search update was February 18, 2014, using the search terms: 'Serine/threonine kinase 15 or STK15 or Aurora-A or AURKA' and 'genetic polymorphism or polymorphisms or variant' and 'digestive system cancer or gastric cancer or colorectal cancer or hepatocellular carcinoma or pancreatic cancer or esophageal cancer'. The search was restricted to humans without language exclusions. Additional studies were identified by a manual search of the references from the original or review articles on this topic.

Inclusion and exclusion criteria. Studies included in this meta-analysis were selected according to the following criteria: i) Studies that evaluated the association between the STK15 polymorphisms (91T $>$ A or $169 \mathrm{G}>\mathrm{A}$ ) and digestive system cancers; ii) studies that had a case-control design; and iii) studies that had a detailed genotype frequency of cases and controls or that had presented sufficient data for this to be calculated from the article text. The major exclusion criteria were i) case-only studies, case reports and review articles; ii) studies without raw data of the STK15 genotype; and iii) repetitive publications.

Data extraction. For each study, the following data were extracted independently by two investigators: The name of the first author, age and gender of the subjects, year of study publication, country of origin, ethnicity, source of controls, genotype methods, number of cases and controls, and the Hardy-Weinberg equilibrium (HWE) in the controls (P-value). The results were compared and disagreements were discussed among all authors and resolved with consensus.

Statistical analysis. The HWE was evaluated for each study using an internet-based HWE calculator (http://ihg.gsf.de/cgi-bin/hw/hwa1.pl) (24). The risk of digestive system cancers associated with the STK15 polymorphisms was estimated for each study by the odds ratio (OR) and $95 \%$ confidence interval (CI). Four different ORs were calculated: The dominant model (variant homozygote + heterozygote versus wild-type homozygote), the recessive model (variant homozygote versus heterozygote + wild-type homozygote), heterozygote comparison (heterozygote versus wild-type homozygote) and homozygote comparison (variant homozygote versus wild-type homozygote). A $\chi^{2}$-test-based Q statistic test was performed to assess the heterogeneity between studies (25). The effect of heterogeneity was also quantified by the $\mathrm{I}^{2}$ test. When a significant $\mathrm{Q}$ test $(\mathrm{P}>0.05)$ or $\mathrm{I}^{2}$ value $<50 \%$ indicated homogeneity across the studies, the fixed effects model was used (26); otherwise, the random effects model was used (27). Stratification analyses on ethnicity and tumor type were subsequently performed. Analysis of sensitivity was performed to evaluate the stability of the results. Finally, potential publication bias was investigated using Begg's funnel plot and Egger's regression test $(28,29) . \mathrm{P}<0.05$ was considered to indicate a statistically significant difference.

All analyses were performed using the Cochrane Collaboration RevMan 5.2 (The Nordic Cochrane Centre, The Cochrane Collaboration, Copenhagen, 2012) and STATA package version 12.0 (Stata Corporation, College Station, TX, USA).

\section{Results}

Study characteristics. The search strategy retrieved 72 potentially relevant studies. According to the inclusion criteria, 14 studies (10-23) with full-text were included in the present meta-analysis and 58 studies were excluded. The flow chart of the study selection is summarized in Fig. 1. Since the study by Ewart-Toland et al (16) included two populations, these populations were treated separately in the current meta-analysis (Tables I and II); as such, there were 15 case-control studies from 14 publications with 7,619 cases and 7,196 controls concerning the 91T $>$ A polymorphism and four studies with 826 cases and 713 controls concerning the $169 \mathrm{G}>\mathrm{A}$ polymorphism. Of the 15 eligible studies, 10 studies $(11,12,14,10,16-19,22)$ were written in English and five studies $(13,15,20,21,23)$ in Chinese; nine studies $(12,13,15,10,18-21,23)$ were conducted on Asian populations, five studies $(11,14,16,17,22)$ on Caucasian populations and one study (16) on a mixed population. Four tumor types were addressed: Six studies $(12,15,10,19-21)$ focused on esophageal cancer; six studies $(14,16,17,22,23)$ on colorectal cancer; two studies $(13,18)$ on gastric cancer and one study (11) on hepatocellular carcinoma. The distribution of genotypes among the controls was consistent with the HWE for all selected studies, with the exception of three $(12,10,21)$.

Quantitative data synthesis. Fifteen studies reported an association between the $91 \mathrm{~T}>\mathrm{A}$ polymorphism and susceptibility to digestive system cancers. Overall, a significantly increased risk was found under the recessive model (OR, 1.19; 95\% CI, 1.07-1.31) (Fig. 2), while no notable associations were observed under the three other models (dominant model: OR, 1.02; 95\% CI, 0.87-1.21; TA versus TT: OR, 0.97; 95\% CI, 0.83-1.14; AA versus TT: OR, 1.12; 95\% CI, 0.89-1.42).

In the subgroup analysis by ethnicity, a significant association was detected in the Asian population under the recessive model (OR, 1.21; 95\% CI, 1.08-1.36) but under the other three models. No association was observed in the Caucasian or mixed populations.

Stratification by tumor type indicated that the 91T $>$ A polymorphism was associated with an increased risk of esophageal and colorectal cancers under the recessive model (OR, 1.19; 95\% CI, 1.03-1.38; and OR, 1.24; 95\% CI, 1.04-1.46; respectively); however, no significant association was detected for gastric cancer. Only one study focused on hepatocellular cancer and the results showed that the STK15 91T>A polymorphism may be a genetic susceptibility factor for hepatocellular carcinoma (Table III).

Four studies reported an association between the $169 \mathrm{G}>\mathrm{A}$ polymorphism and the risk of digestive system cancers. The 
Table I. Characteristics of studies included in the meta-analysis.

A, Studies on the $91 \mathrm{~T}>\mathrm{A}$ polymorphism

\begin{tabular}{lllllll}
\hline First author (ref.) & Year & Country & Ethnicity & Tumor type & $\begin{array}{c}\text { Source of } \\
\text { controls }\end{array}$ & $\begin{array}{c}\text { Genotype } \\
\text { methods }\end{array}$ \\
\hline Akkiz (11) & 2010 & Turkey & Caucasian & Hepatocellular & HB & PCR-RFLP \\
Chava (12) & 2011 & India & Asian & Esophageal & NR & PCR \\
Chen L (13) & 2005 & China & Asian & Gastric & HB & PCR-RFLP \\
Chen JY (14) & 2007 & USA & Caucasian & Colorectal & HB & Direct sequencing \\
Chen XB (15) & 2009 & China & Asian & Esophageal & PB & PCR-RFLP \\
Ewart-Toland (16) & $2005 a$ & USA & Mixed & Colorectal & PB & PCR-RFLP \\
Ewart-Toland (16) & $2005 b$ & Scotland & Caucasian & Colorectal & PB & PCR-RFLP \\
Hienonen (17) & 2006 & Finland & Caucasian & Colorectal & PB & Direct sequencing \\
Ju (18) & 2006 & South Korea & Asian & Gastric & HB & Mass ARRAY \\
Kimura (10) & 2005 & Japan & Asian & Esophageal & HB & PCR \\
Miao (19) & 2004 & China & Asian & Esophageal & PB & PCR-RFLP \\
Sang (20) & 2012 & China & Asian & Esophageal & HB & MALDI-TOF MS \\
Wang (21) & 2007 & China & Asian & Esophageal & PB & PCR-RFLP \\
Webb (22) & 2006 & UK & Caucasian & Colorectal & PB & Illuminasentric \\
Zhang (23) & 2006 & China & Asian & Colorectal & PB & bead array
\end{tabular}

$\mathrm{B}$, Studies on the $169 \mathrm{G}>\mathrm{A}$ polymorphism

\begin{tabular}{lllllll}
\hline First author (ref.) & Year & Country & Ethnicity & Tumor type & $\begin{array}{c}\text { Source of } \\
\text { controls }\end{array}$ & $\begin{array}{c}\text { Genotype } \\
\text { methods }\end{array}$ \\
\hline Chen L (12) & 2005 & China & Asian & Gastric & HB & PCR-RFLP \\
Chen JY (13) & 2007 & USA & Caucasian & Colorectal & HB & Direct sequencing \\
Ju (17) & 2006 & South Korea & Asian & Gastric & HB & Mass ARRAY \\
Kimura (18) & 2005 & Japan & Asian & Esophageal & HB & PCR \\
\hline
\end{tabular}

NR, not reported; HB, hospital-based; PB, population-based; MALDI-TOF MS, matrix-assisted laser desorption/ionization time of flight mass spectrometry; PCR-RFLP, polymerase chain reaction-restriction fragment length polymorphism; ref., reference.

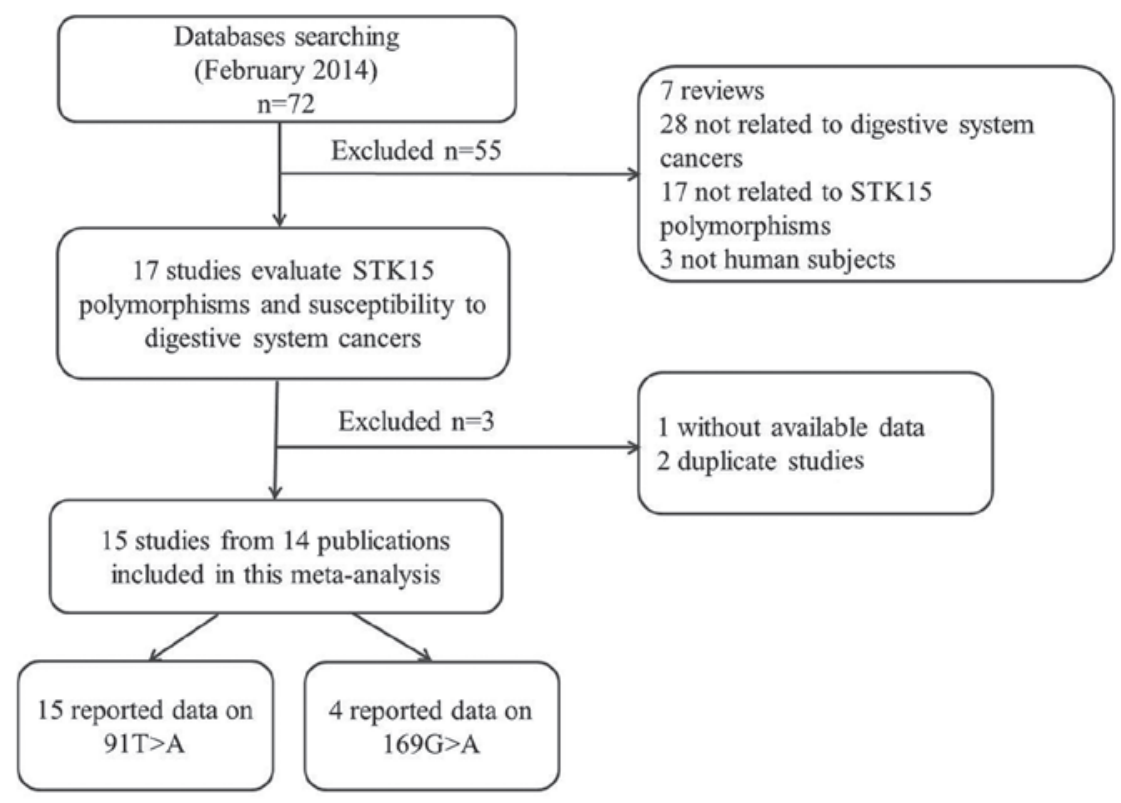

Figure 1. Flow chart showing the study selection procedure. STK15, serine/threonine kinase 15. 

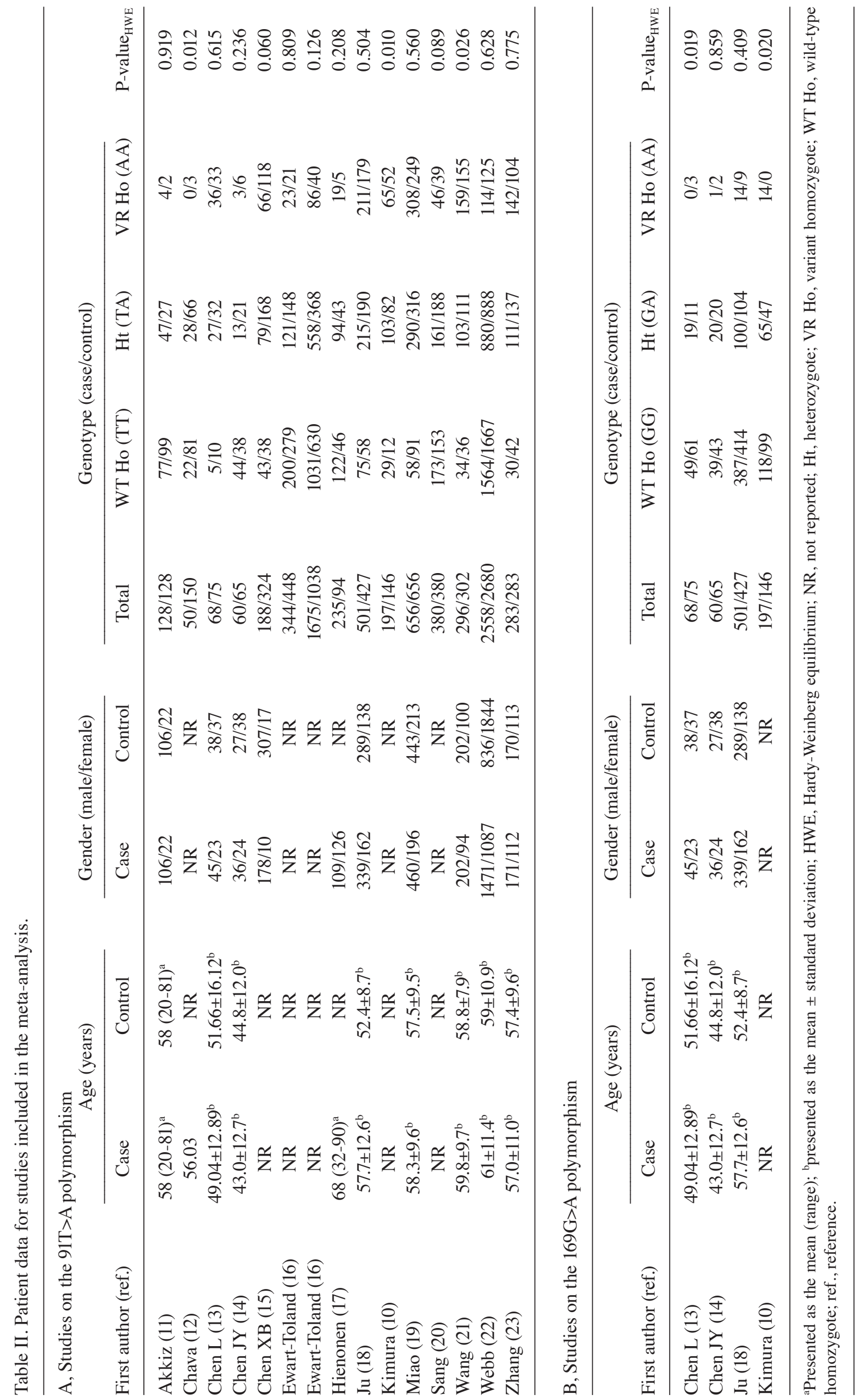


\begin{tabular}{|c|c|c|c|c|c|c|c|c|c|}
\hline Study or Subgroup & $\begin{array}{l}\text { Case } \\
\text { Events }\end{array}$ & Total & $\begin{array}{l}\text { Contr } \\
\text { Events }\end{array}$ & $\begin{array}{l}\text { rol } \\
\text { Total }\end{array}$ & Weight & $\begin{array}{c}\text { Odds Ratio } \\
\text { M-H, Fixed, } 95 \% \mathrm{Cl}\end{array}$ & $\begin{array}{r}\text { Odds } \\
\text { M-H, Fix }\end{array}$ & $\begin{array}{l}\text { s Ratio } \\
\text { ed, } 95 \%, \mathrm{Cl}\end{array}$ & \\
\hline Akkiz 2010 & 4 & 128 & 2 & 128 & $0.3 \%$ & $2.03[0.37-11.30]$ & & & \\
\hline Chava 2011 & 0 & 50 & 3 & 150 & $0.2 \%$ & $0.42[0.02-8.22]$ & & & \\
\hline Chen JY 2007 & 3 & 60 & 6 & 65 & $0.8 \%$ & $0.52[0.12-2.17]$ & & & \\
\hline Chen L 2005 & 36 & 68 & 33 & 75 & $2.1 \%$ & $1.43[0.74-2.77]$ & & & \\
\hline Chen XВ 2009 & 66 & 188 & 118 & 324 & $7.9 \%$ & $0.94[0.65-1.37]$ & & & \\
\hline Ewart-Toland 2005a & 23 & 344 & 21 & 448 & $2.4 \%$ & $1.46[0.79-2.68]$ & & & \\
\hline Ewart-Toland 2005b & 86 & 1675 & 40 & 1038 & $6.6 \%$ & $1.35[0.92-1.98]$ & & - & \\
\hline Hienonen 2006 & 19 & 235 & 5 & 94 & $0.9 \%$ & $1.57[0.57-4.32]$ & & & \\
\hline Ju 2006 & 211 & 501 & 179 & 427 & $15.8 \%$ & $1.01[0.78-1.31]$ & & - & \\
\hline Kimura 2005 & 65 & 197 & 52 & 146 & $5.7 \%$ & $0.89[0.57-1.40]$ & & & \\
\hline Miao 2004 & 308 & 656 & 249 & 656 & $18.6 \%$ & $1.45[1.16-1.80]$ & & - & \\
\hline Sang 2012 & 46 & 380 & 39 & 380 & $4.8 \%$ & $1.20[0.77-1.89]$ & & 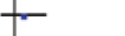 & \\
\hline Wang 2007 & 159 & 296 & 155 & 302 & $10.0 \%$ & $1.10[0.80-1.52]$ & & & \\
\hline Webb 2006 & 114 & 2558 & 125 & 2680 & $16.5 \%$ & $0.95[0.74-1.24]$ & & & \\
\hline Zhang 2006 & 142 & 283 & 104 & 283 & $7.3 \%$ & $1.73[1.24-2.42]$ & & - & \\
\hline Total $(95 \% \mathrm{Cl})$ & & 7619 & & 7196 & $100.0 \%$ & $1.19[1.07-1.31]$ & & 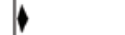 & \\
\hline Total events & 1282 & & 1131 & & & & & & \\
\hline $\begin{array}{l}\text { Heterogeneity: } \mathrm{Ch}^{2}= \\
\text { Test for overall effect }\end{array}$ & $\begin{array}{l}9.04, \mathrm{df}= \\
=3.35 \mathrm{~K}\end{array}$ & $\begin{array}{l}=14(P \\
P=0.0\end{array}$ & $\begin{array}{l}=0.16) ; 1^{2} \\
008)\end{array}$ & $2=26 \%$ & & & 0.020 .1 & 1 & $10 \quad 50$ \\
\hline
\end{tabular}

Figure 2. Forest plots for the association between the serine/threonine kinase 15 gene $91 \mathrm{~T}>\mathrm{A}$ polymorphism and digestive system cancers risk under a recessive model. CI, confidence interval.

combined results based on all the studies revealed no significant associations among the studies with any of the genetic models (dominant model: OR, 1.02; 95\% CI, 0.82-1.28; recessive model: OR, 1.27; 95\% CI, 0.25-6.49; GA versus GG: OR, 1.13; 95\% CI, 0.90-1.43; AA versus GG: OR, 1.45; $95 \%$ CI, 0.29-7.22). In the subgroup analysis by ethnicity, similar results were demonstrated in the Asian and Caucasian populations (Table III).

Heterogeneity and sensitivity analyses. Substantial heterogeneities were observed among the studies for the association between the risk of digestive system cancers and the $91 \mathrm{~T}>\mathrm{A}$ (dominant model: $\mathrm{I}^{2}=68 \%, \mathrm{P}<0.0001$; TA versus TT: $\mathrm{I}^{2}=63 \%$, $\mathrm{P}=0.0005$; AA versus TT: $\left.\mathrm{I}^{2}=58 \%, \mathrm{P}=0.002\right)$ and $169 \mathrm{G}>\mathrm{A}$ (recessive model: $\mathrm{I}^{2}=58 \%, \mathrm{P}=0.07$; AA versus $\mathrm{TT}$ : $\mathrm{I}^{2}=56 \%$, $\mathrm{P}=0.08) \mathrm{STK} 19$ polymorphisms. The source of the heterogeneity for the genetic model comparisons by ethnicity and tumor site was subsequently analyzed. For the $91 \mathrm{~T}>\mathrm{A}$ polymorphism, the heterogeneity was partially decreased or removed in colorectal and gastric cancers and Caucasian populations; however, significant heterogeneity remained for esophageal cancer and Asian populations. For the $169 \mathrm{G}>\mathrm{A}$ polymorphism, the heterogeneity significantly decreased when the study by Kimura et al (10) was excluded from the analysis. A sensitivity analysis was performed to evaluate the stability of the results. Since the statistical significance of the results did not change when any single study was omitted, the stability of the results was confirmed.

Publication bias. Begg's funnel plot and Egger's tests were used to address potential publication bias in the available literature. The shape of the funnel plots did not show any evidence of funnel plot asymmetry (data not shown). Egger's test also demonstrated that there was no statistical significance in the evaluation of publication bias (dominant model, $\mathrm{P}=0.991$; TA versus TT, $\mathrm{P}=0.721$; AA versus TT, $\mathrm{P}=0.925$; recessive model, $\mathrm{P}=0.835)$.

\section{Discussion}

STK15, a member of the Aurora family, plays a vital role in bipolar mitotic spindle formation and regulates chromosome segregation in mammalian cells (30). It has been reported that STK15 is overexpressed in numerous types of cancer, including colorectal, pancreatic, breast and prostate $(6,7,31,32)$. Although the mechanism remains unclear, it is believed that the polymorphism may partially affect STK15 expression and therefore modify its function. Ewart-Toland et al (8) suggested that the STK15 91T >A polymorphism $(\mathrm{T} \rightarrow \mathrm{A})$ variant changed the activity of the STK15 box 1, leading to an inhibition of p53 binding and the decreased degradation of STK15. It was further suggested that the stabilized overexpression of STK15 led to centrosome amplification, improper cytokinesis, chromosomal instability and the promotion of tumorigenesis (8). To date, a number of studies have investigated the association between STK15 polymorphisms and the risk of cancers, particularly cancers of the digestive system (10-23); however, the results have been inconsistent. In a study from Turkey, Akkiz et al (11) reported that the STK15 91T>A polymorphism may be a genetic susceptibility factor for hepatocellular carcinoma. Similarly, Hienonen et al (17) observed that the STK15 91T>A polymorphism was a low penetrance colorectal cancer susceptibility factor in Finnish populations; however, Webb et al (22) suggested that there was no association between the polymorphism and colorectal cancer susceptibility based on their results. With regard to the $169 \mathrm{G}>\mathrm{A}$ polymorphism, Ju et al (18) reported that the $169 \mathrm{G}>\mathrm{A}$ polymorphism in the STK15 gene was associated with the progression of gastric cancer; however, in a study from China, Chen (13) failed to detect any association between the $169 \mathrm{G}>\mathrm{A}$ polymorphism and the risk of gastric cancer.

Recently, two meta-analyses $(33,34)$ evaluated the association between the STK15 91T>A polymorphism and risk of cancer, and reported that the STK15 91T>A polymorphism may be a risk factor for cancer. In comparison, the present 
study conducted a comprehensive literature search of different databases and included several additional studies. Furthermore, the association between the $169 \mathrm{G}>\mathrm{A}$ polymorphism and the risk of digestive system cancers was explored. In the current meta-analysis, 15 studies were pooled to examine the association between the two STK15 polymorphisms and risk of digestive system cancers. The results demonstrated that there was a significant association between the STK15 91T>A polymorphism and the risk of digestive system cancers.

In the subgroup analysis by ethnicity, there was a significant association in Asian descent, but not in Caucasian and mixed populations. Different genetic backgrounds and environmental exposures among the different ethnic groups may contribute to this discrepancy (35). When stratified by tumor type, the 91T $>$ A polymorphism was associated with an increased risk of esophageal and colorectal cancers, but not gastric cancer. Only one study focused on hepatocellular carcinoma and the results revealed that the STK15 91T>A polymorphism may be a genetic susceptibility factor for hepatocellular carcinoma; however, since only a few studies on gastric cancer and hepatocellular carcinoma were included, these results should be interpreted with caution, and further studies are required.

No significant association was found between the $169 \mathrm{G}>\mathrm{A}$ polymorphism and the risk of digestive system cancers in any of the genetic models. When stratified according to ethnicity, similar results were observed in Asian and Caucasian populations. This lack of association may have been due to the limited literature (only four studies) in the present meta-analysis. The conclusions should therefore be considered sensibly. Furthermore, cancer is a multi-factorial disease that results from complex interactions between a number of environmental and genetic factors (gene-gene or gene-environment). Not all of the studies included, however, analyzed the same environmental or genetic factors and, due to lack of individual data in the present review, more detailed analyses, such as analyses of joint effects with other risk factors or gene-gene or gene-environment interactions, were not able to be performed.

Heterogeneity is a potential problem when interpreting the results of all meta-analyses (36). In the current meta-analysis, heterogeneity was observed in the overall comparison for certain genetic models. When stratified by ethnicity and tumor site, the heterogeneity was partially decreased or removed in colorectal and gastric cancers and Caucasian populations; however, heterogeneity remained for esophageal cancer and Asian populations. For the $169 \mathrm{G}>\mathrm{A}$ polymorphism, the heterogeneity significantly decreased when the study by Kimura et al (10) was excluded from analysis. These results suggest that the ethnic difference, different tumor types and particular study type may be the source of heterogeneity in the present meta-analysis. When sensitivity analyses were conducted by successively excluding one study, the estimated pooled OR changed little, strengthening the results from the meta-analysis. Furthermore, no publication bias was observed, highlighting the possibility of true results.

The current meta-analysis has limitations that require acknowledgement. Firstly, due to incomplete raw data or publication limitations, certain relevant studies were unable to be included in the present analysis. Secondly, the results were based on unadjusted estimates, which may cause serious confounding bias. Thirdly, the data from the European populations were relatively small and significant heterogeneity was observed in certain models, which may have resulted in failure to confirm marginal associations.

In conclusion, the present meta-analysis suggests that the STK15 gene $91 \mathrm{~T}>\mathrm{A}$ polymorphism, but not the $169 \mathrm{G}>\mathrm{A}$ polymorphism, may be a risk factor for digestive system cancers, particularly for esophageal and colorectal cancers.

\section{References}

1. Jemal A, Center MM, DeSantis C and Ward EM: Global patterns of cancer incidence and mortality rates and trends. Cancer Epidemiol Biomarkers Prev 19: 1893-1907, 2010.

2. Pharoah PD, Dunning AM, Ponder BA and Easton DF: Association studies for finding cancer-susceptibility genetic variants. Nat Rev Cancer 4: 850-860, 2004.

3. Risch N: The genetic epidemiology of cancer: interpreting family and twin studies and their implications for molecular genetic approaches. Cancer Epidemiol Biomarkers Prev 10: 733-741, 2001.

4. Nigg EA: Centrosome aberrations: cause or consequence of cancer progression? Nat Rev Cancer 2: 815-825, 2002.

5. Zhou H, Kuang J, Zhong L, et al: Tumour amplified kinase STK15/BTAK induces centrosome amplification, aneuploidy and transformation. Nat Genet 20: 189-193, 1998.

6. Bischoff JR, Anderson L, Zhu Y, et al: A homologue of Drosophila aurora kinase is oncogenic and amplified in human colorectal cancers. EMBO J 17: 3052-3065, 1998.

7. Li D, Zhu J, Firozi PF, et al: Overexpression of oncogenic STK15/BTAK/Aurora A kinase in human pancreatic cancer. Clin Cancer Res 9: 991-997, 2003.

8. Ewart-Toland A, Briassouli P, de Koning JP, et al: Identification of Stk6/STK15 as a candidate low-penetrance tumor-susceptibility gene in mouse and human. Nat Genet 34: 403-412, 2003.

9. Venter JC, Adams MD, Myers EW, et al: The sequence of the human genome. Science 291: 1304-1351, 2001.

10. Kimura MT, Mori T, Conroy J, Nowak NJ, Satomi S, Tamai K and Nagase H: Two functional coding single nucleotide polymor $\neg$ phisms in STK15 (Aurora A) coordinately increase esophageal cancer risk. Cancer Res 65: 3548-3554, 2005.

11. Akkiz H, Bayram S, Bekar A, Akgöllü E and Ozdil B: Relationship between functional polymorphism in the Aurora A gene and susceptibility of hepatocellular carcinoma. J Viral Hepat 17: 668-674, 2010.

12. Chava S, Mohan V, Pasupuleti N, et al: Evaluation of Aurora-A gene polymorphism and esophageal cancer risk in a South Indian population. Genet Test Mol Biomarkers 15: 185-189, 2011.

13. Chen L: Genetic polymorphisms and disease risks: STK15 gene and risk for gastric cancer, MMP9 gene and risk for thoracic aortic aneurysm and thoracic aortic dissection (unpublished $\mathrm{PhD}$ thesis). China Medical University: 2005 (In Chinese).

14. Chen J, Sen S, Amos CI, Wei C, Jones JS, Lynch P and Frazier ML: Association between Aurora-A kinase polymorphisms and age of onset of hereditary nonpolyposis colorectal cancer in a Caucasian population. Mol Carcinog 46: 249-256, 2007.

15. Chen XB, Chen GL, Liu JN, Yang JZ, Yu DK, Lin DX and Tan W: Genetic polymorphisms in STK15 and MMP-2 associated susceptibility to esophageal cancer in Mongolian population. Zhonghua Yu Fang Yi Xue Za Zhi 43: 559-564, 2009 (In Chinese).

16. Ewart-Toland A, Dai Q, Gao YT, et al: Aurora-A/STK15 $\mathrm{T}+91 \mathrm{~A}$ is a general low penetrance cancer susceptibility gene: a meta-analysis of multiple cancer types. Carcinogenesis 26: 1368-1373, 2005

17. Hienonen T, Salovaara R, Mecklin JP, Järvinen H, Karhu A and Aaltonen LA: Preferential amplification of AURKA 91A (Ile31) in familial colorectal cancers. Int J Cancer 118: 505-508, 2006.

18. Ju H, Cho H, Kim YS, et al: Functional polymorphism 57Val>Ile of aurora kinase A associated with increased risk of gastric cancer progression. Cancer Lett 242: 273-279, 2006.

19. Miao X, Sun T, Wang Y, Zhang X, Tan W and Lin D: Functional STK15 Phe31Ile polymorphism is associated with the occurrence and advanced disease status of esophageal squamous cell carcinoma. Cancer Res 64: 2680-2683, 2004.

20. Sang YH, Gu HY, Ding GW, Yang WT, Chen SC and Chen YB: Association between the esophageal cancer susceptibility and the STK15 T+91A polymorphism. Zhonghua Shi Yan Wai Ke Za Zhi 29: 1220, 2012 (In Chinese). 
21. Wang N, Wang GY, Guo W, Dong XJ and Li Y: Study on the association between STK15 Phe31Ile polymorphisms and esophageal squamous cell carcinoma. Zhonghua Liu Xing Bing Xue Za Zhi 28: 394-397, 2007 (In Chinese).

22. Webb EL, Rudd MF and Houlston RS: Case-control, kin-cohort and meta-analyses provide no support for STK15 F31I as a low penetrance colorectal cancer allele. Br J Cancer 95: 1047-1049, 2006.

23. Zhang WJ, Miao XP, Sun T, et al: Association between genetic polymorphism in STK15 and risk of colorectal cancer in a Chinese population. Zhonghua Zhong Liu Za Zhi 28: 43-46, 2006 (In Chinese)

24. Institute of Human Genetics. HWE calculator. (http://ihg.gsf.de/cgi-bin/hw/hwal.pl). Accessed February 20, 2014

25. Lau J, Ioannidis JP and Schmid CH: Quantitative synthesis in systematic reviews. Ann Intern Med 127: 820-826, 1997.

26. Mantel N and Haenszel W: Statistical aspects of the analysis of data from retrospective studies of disease. J Natl Cancer Inst 22: 719-748, 1959

27. DerSimonian R and Laird N: Meta-analysis in clinical trials. Control Clin Trials 7: 177-188, 1986.

28. Begg CB and Mazumdar M: Operating characteristics of a rank correlation test for publication bias. Biometrics 50: 1088-1101, 1994.

29. Egger M, Davey Smith G, Schneider M and Minder C: Bias in meta-analysis detected by a simple, graphical test. BMJ 315: 629-634, 1997.
30. Egan KM, Newcomb PA, Ambrosone CB, et al: STK15 polymorphism and breast cancer risk in a population-based study. Carcinogenesis 25: 2149-2153, 2004.

31. Tanaka T, Kimura M, Matsunaga K, Fukada D, Mori H and Okano Y: Centrosomal kinase AIK1 is overexpressed in invasive ductal carcinoma of the breast. Cancer Res 59: 2041-2044, 1999.

32. Buschhorn HM, Klein RR, Chambers SM, Hardy MC, Green S, Bearss D and Nagle RB: Aurora-A over-expression in high-grade PIN lesions and prostate cancer. Prostate 64: 341-346, 2005.

33. Tang W, Qiu H, Ding H, Sun B, Wang L, Yin J and Gu H: Association between the STK15 F31I polymorphism and cancer susceptibility: a meta-analysis involving 43,626 subjects. PLoS One 8: e82790, 2013.

34. Xu L, Zhou X, Jiang F, Xu L and Yin R: STK15 rs2273535 polymorphism and cancer risk: a meta-analysis of 74,896 subjects. Cancer Epidemiol 38: 111-117, 2014

35. Hirschhorn JN, Lohmueller K, Byrne E and Hirschhorn K: A comprehensive review of genetic association studies. Genet Med 4: 45-61, 2002.

36. Boccia S, De Feo E, Galli P, Gianfagna F, Amore R and Ricciardi G: A systematic review evaluating the methodological aspects of meta-analyses of genetic association studies in cancer research. Eur J Epidemiol 25: 765-775, 2010. 